\title{
Deciphering how the viscoelastic properties of mussel-inspired metal- coordinate transiently crosslinked gels dictate their tack behavior
}

\author{
Erica Lai ${ }^{1}$, Bavand Keshavarz ${ }^{2}$, and Niels Holten-Andersen ${ }^{1 *}$ \\ ${ }^{1}$ Department of Materials Science and Engineering, ${ }^{2}$ Department of Mechanical Engineering \\ Massachusetts Institute of Technology \\ Cambridge, Massachusetts, 02139, USA \\ *holten@mit.edu
}

\section{Table of Contents}

S1. Theoretical Prediction for the Temporal Evolution of the Tack Force - The Full Derivation.

Figure S1. Representative frequency sweep for one of the 4PEG-His $+\mathrm{Ni}^{2+}$ hydrogels studied.

Figure S2. The average $G_{p}$ and $\tau_{c}$ for (a) $10 \mathrm{wt} \%$ and (b) $5 \mathrm{wt} \%$ PPEG-His $+\mathrm{Ni}^{2+}$ gels.................... 55

Figure S3. A schematic depicting the three qualitative categories of sample behavior. S6

Figure S4. $\sigma_{\max } / G_{p}$ vs. Wi with the cavitation cases included. S7

Figure S5. For non-cavitation cases of tack tests on 4PEG-His $+\mathrm{Ni}^{2+}$ gels, [EDV graphs]. S8 


\section{S1. Theoretical Prediction for the Temporal Evolution of the Tack Force - The Full Derivation}

Assuming that the flow in the thin gap is dominated by viscous effects and the pressure gradient under the plate, as shown in Eqn. (1), is mainly balanced by the gradient of viscous stresses (similar to the squeeze flow which is also known as Stefan's flow ${ }^{1}$ ).

$$
\begin{aligned}
& (r): \frac{\partial P}{\partial r}=\frac{\partial \tau_{r z}}{\partial z} \\
& (z): \frac{\partial P}{\partial z}=\frac{\partial \tau_{z z}}{\partial z}
\end{aligned}
$$

For a Newtonian liquid (with constant viscosity) the stress components in Eqn. (1) can be connected to the corresponding deformation rates of the flow. This enables us to solve for the deformation rates and the velocity flowfield. Similar to the work of Spiegelberg et al. ${ }^{2}$, for viscous Newtonian liquids, the temporal evolution of the flowfield can be expressed in terms of geometrical position of fluid elements and the plate height profile $(h(t))$ :

$$
\begin{gathered}
v_{r}(r, z, t)=-3 \dot{h}(t)\left(\frac{r}{h(t)}\right)\left(\frac{z}{h(t)}\right)\left(1-\frac{z}{h(t)}\right) \\
v_{z}(r, z, t)=\dot{h}(t)\left(\frac{z}{h(t)}\right)^{2}\left(3-2 \frac{z}{h(t)}\right)
\end{gathered}
$$

This leads to an expression for the change in the radius of the filament at the mid-plane (i.e. where $z=$ $h(t) / 2)$ :

$$
R_{\text {mid }} / R_{0}=\left(h(t) / h_{0}\right)^{-3 / 4}
$$

It has been shown that the described Newtonian kinematics (Equations 2 and 3 ) is fairly accurate for even viscoelastic liquids (see thesis by Jaishankar ${ }^{3}$ ). The approximate kinematics can be coupled with any linear constitutive equations to provide an integral form for the fluid stresses:

$$
\tau(t)=\int_{-\infty}^{t} G\left(t-t^{\prime}\right) \dot{\gamma}\left(t^{\prime}\right) d t^{\prime}
$$

where $G(t)$ is the relaxation modulus of the material. Inserting the kinematics, Eqn. (2), into this integral form, Eqn. (4), we can describe the stress field in the integral form. The fluid stresses are related to the pressure gradient through the Stefan's flow approximation, which is expressed in Eqn. (1). Integrating the mentioned expression in space leads to the following solution for the pressure field:

where

$$
P(r, z, t)=\left[3\left(r^{2}-R_{0}^{2}\right)+12 z(h-z)\right] \mathbb{H}(t)
$$

$$
\mathbb{H}(t) \equiv \int_{-\infty}^{t} \frac{G\left(t-t^{\prime}\right) \dot{h}\left(t^{\prime}\right)}{h^{3}\left(t^{\prime}\right)} d t^{\prime}
$$

The pressure field has both spatial and temporal variations. To find the acting suction force on the moving plate, the pressure distribution on the plate is evaluated and integrated at each given time. The suction force can be described as a function of the plate height: 


$$
F(t)=3 \pi R_{0}^{4} \mathbb{H}(t)\left[\left(\frac{h}{h_{0}}\right)^{-3 / 2}-\frac{1}{2}\left(\frac{h}{h_{0}}\right)^{-3}\right]
$$

We can now replace the relaxation modulus in Eqn. (6) with the corresponding exponential function for the Maxwell model $\left(G(t)=G_{0} \exp \left(-t / \tau_{c}\right)\right.$ where $G_{0}$ and $\tau_{c}$ are respectively the plateau modulus and the relaxation time of the material). This can be simplified and the final expression for the temporal evolution of the suction force can be written as:

$$
\frac{F(t)}{G_{p} \pi R_{0}^{2}}=3\left(\frac{R_{0}}{h_{0}}\right)^{2} \frac{W i}{1-2 W i}\left[\exp \left(-2 W i \frac{t}{\tau_{c}}\right)-\exp \left(-\frac{t}{\tau_{c}}\right)\right]\left[\exp \left(-2 W i \frac{t}{3 \tau_{c}}\right)-\frac{1}{2} \exp \left(-3 W i \frac{t}{\tau_{c}}\right)\right]
$$

The geometrical parameters $R_{0}$ and $h_{0}$ represent the plate radius and the initial gap height. This relationship simply shows that the maximum adhesion force is set by a combination of the linear rheological properties (such as $G_{p}$ ) and geometrical factors (such as $R_{0} / h_{0}$ ). However, it also suggests that the maximum adhesion force should depend on the Weissenberg number $\left(W i \equiv \tau_{c} \dot{\varepsilon}\right)$ or in other words on the applied stretch rate. The maximum of this expression can be easily calculated at different Weissenberg numbers (using a simple numerical code in Matlab) and the suggested model can be compared with the experimental measurements in Figure 4.

\section{References}

(1) Stefan, J. Versuche Über Die Scheinbare Adhäsion. Ann. Phys. 1875, 230 (2), 316-318. https://doi.org/10.1002/andp.18752300213.

(2) Spiegelberg, S. H.; Ables, D. C.; McKinley, G. H. The Role of End-Effects on Measurements of Extensional Viscosity in Filament Stretching Rheometers. J. Nonnewton. Fluid Mech. 1996, 64 (23), 229-267. https://doi.org/10.1016/0377-0257(96)01439-5.

(3) Jaishankar, A. The Linear and Nonlinear Rheology of Multiscale Complex Fluids, Massachusetts Institute of Technology, 2014. 


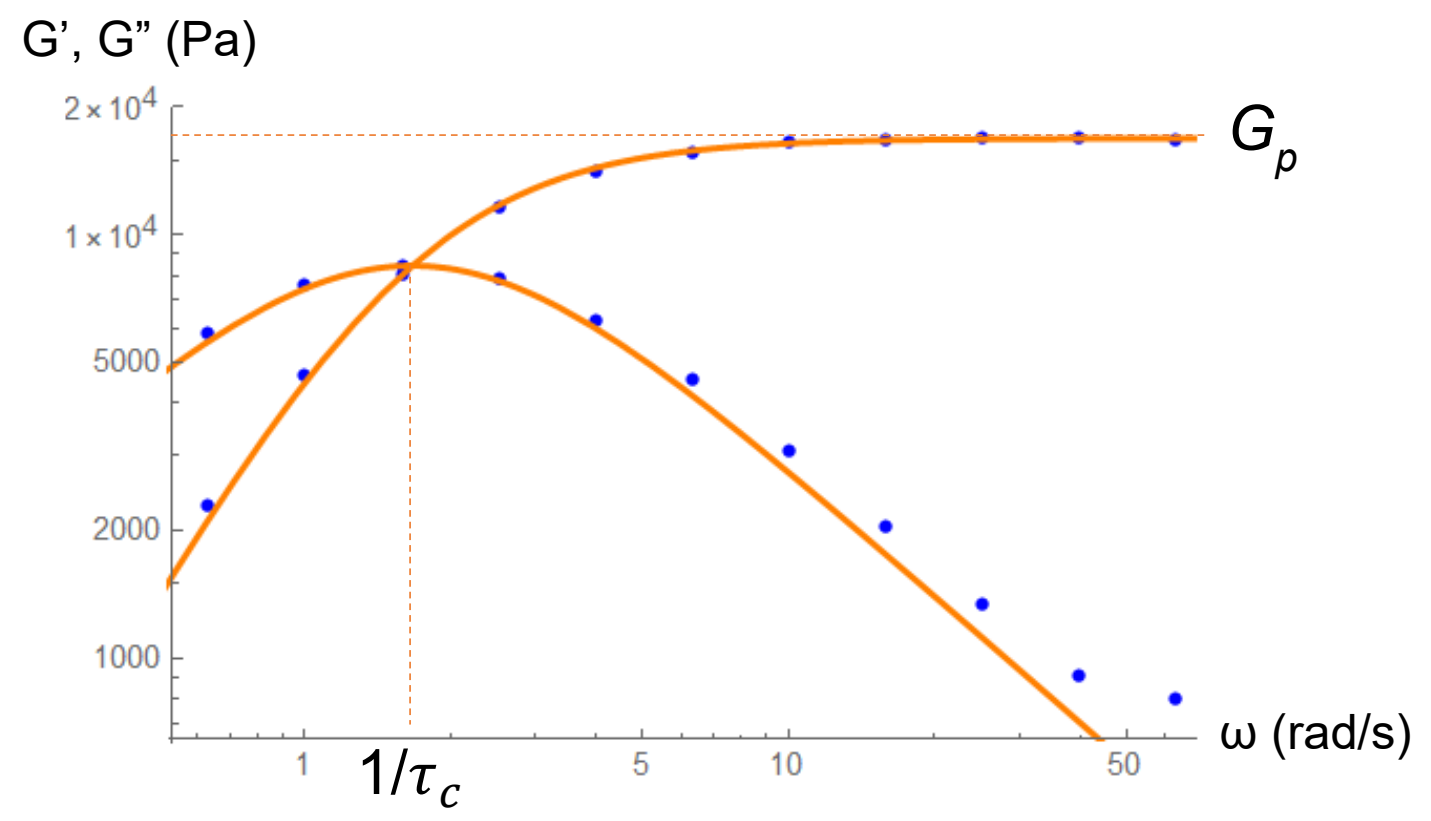

Figure S1. Representative frequency sweep for one of the 4PEG-His + $\mathrm{Ni}^{2+}$ hydrogels studied; the points are experimental data, while the solid line is the single-mode Maxwellian fit. From the fit, the plateau modulus $\left(G_{p}\right)$ and the characteristic relaxation time $\left(\tau_{c}\right)$ were obtained for each hydrogel sample. 
Supporting Information
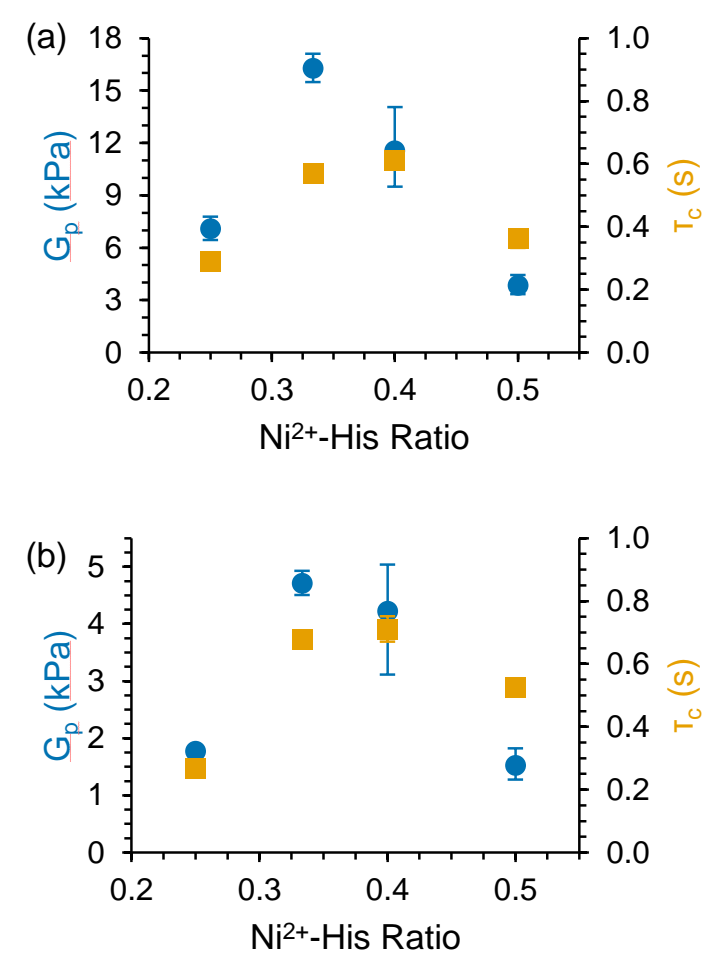

Figure S2. The average $\mathrm{G}_{p}$ and $\tau_{c}$ for (a) $10 \mathrm{wt} \%$ and (b) $5 \mathrm{wt} \% 4 \mathrm{PEG}-\mathrm{His}+\mathrm{Ni}^{2+}$ gels, based on their metalligand ratio. 


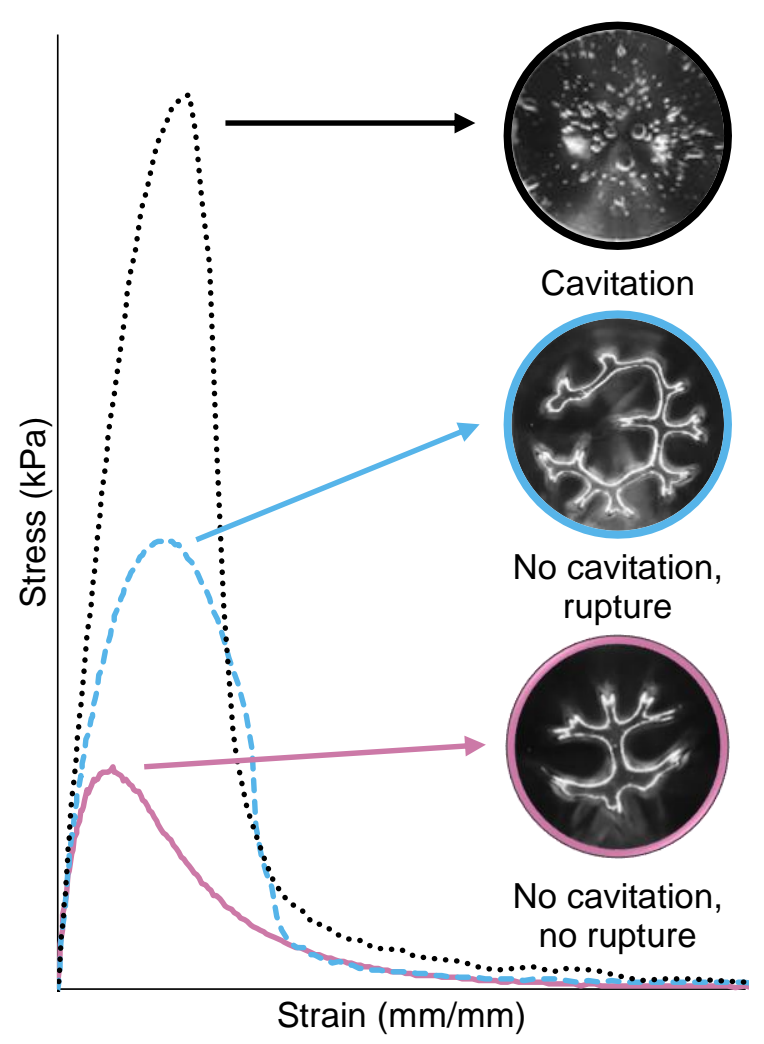

Figure S3. A schematic depicting the three qualitative categories of sample behavior during a tack test regarding cavitation and rupture, and the corresponding stress-strain curve shape. The images are representative snapshots of samples taken from beneath the glass substrate. 


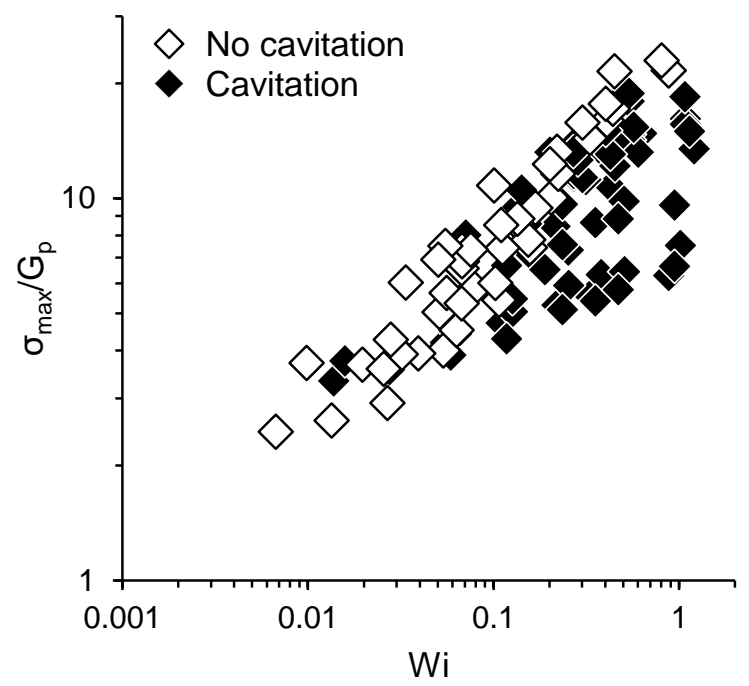

Figure S4. $\sigma_{\max } / G_{p}$ vs. Wi with the cavitation cases included (filled symbols) and plotted on log-log axes. Samples that undergo cavitation do not follow the theoretical model plotted in Figure 4 because the onset of cavitation reduces $\sigma_{\max }$. 


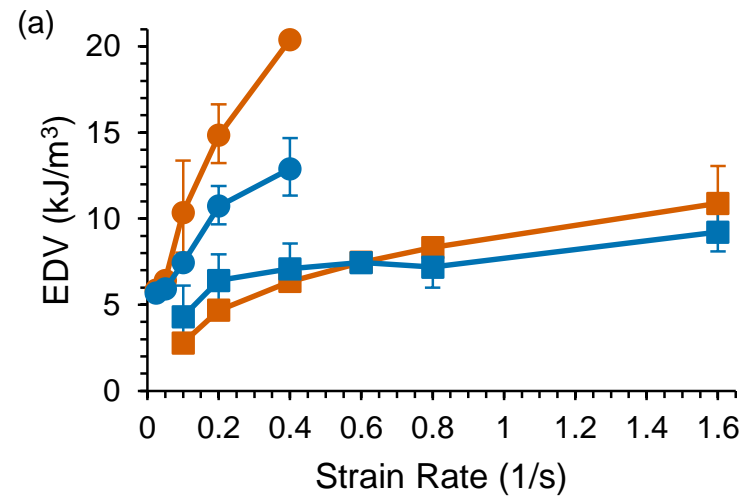

(b)

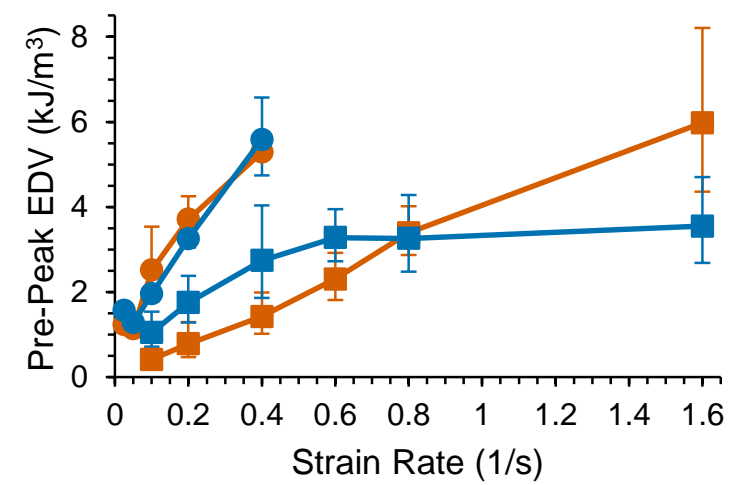

(d)

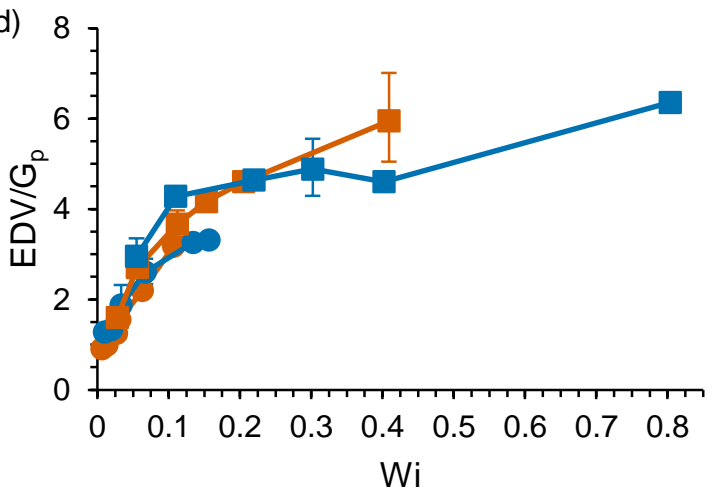

(c)

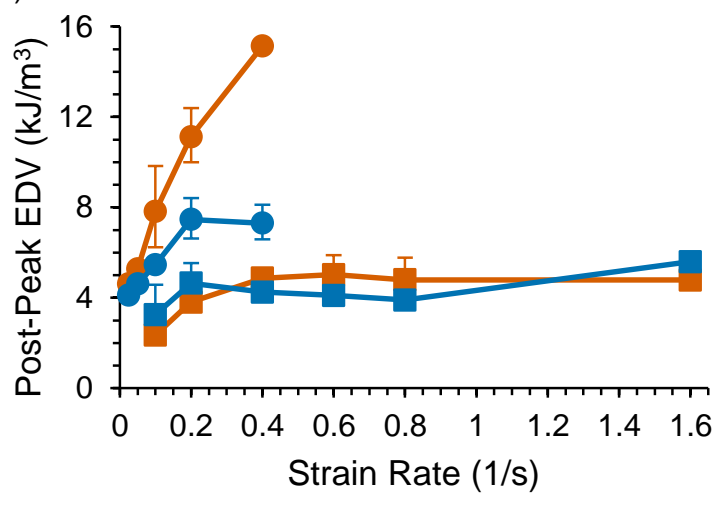

- $10 w t \% \square 5 w t \%$

$\mathrm{Ni}^{2+-}$-His ratio: $0.25,0.5$

Figure S5. For non-cavitation cases of tack tests on 4PEG-His $+\mathrm{Ni}^{2+}$ gels, counterclockwise from top left: (a) total EDV, (b) pre-peak EDV, and (c) post-peak EDV vs. strain rate; (d) total EDV vs. Wi. 